\title{
Institutional Health Care for the Elderly in North-eastern Thailand
}

\author{
Ajchara Pornsema ${ }^{1}$, Kosit Phaengsoi ${ }^{1}$ \& Chanita Summat ${ }^{1}$ \\ ${ }^{1}$ The Faculty of Cultural Science, Mahasarakham University, Khamriang Sub-District, Kantarawichai District, \\ Maha Sarakham Province, Thailand \\ Correspondence: Ajchara Pornsema, The Faculty of Cultural Science, Mahasarakham University, Khamriang \\ Sub-District, Kantarawichai District, Maha Sarakham Province 44150, Thailand. E-mail: ajchsema139 \\ @hotmail.com
}

Received: April 29, 2014 Accepted: May 28, 2014 Online Published: June 12, 2014

doi:10.5539/ach.v6n2p97 URL: http://dx.doi.org/10.5539/ach.v6n2p97

\begin{abstract}
This qualitative research aims to assess the state of institutional health care in North-eastern Thailand. The investigation was conducted from October 2012 to October 2013. Results found that a number of elderly people are neglected by their families. The majority have difficulty moving, poor health and emotional problems. Everyday lifestyle problems are caused by inadequate exercise. Caregivers must be dedicated. Social activities are provided for elderly people, such as Old People's Day, Mother's Day and New Year's Day. Most elderly people receive a pension and some supplement their income through other sources. Elderly people have a low income and a high cost of living so are required to work to support themselves. The government and private sector both support a variety of activities to promote the healthcare of elderly people in North-eastern Thailand.
\end{abstract}

Keywords: social science, healthcare, nursing home, Isan

\section{Introduction}

In the past, elderly people were respected as pillars of the community. Traditionally all people over the age of 60 were considered to be in the 'elderly' bracket and were cared for by their extended families (Sasat, 2006). However, the numbers of people living in extended families is decreasing and, given the advancements in medical technology and the increase of life expectancy, the lower age limit for this group is becoming disputed (College of Population Studies, 2007). While nowadays there is a greater realisation of the value of the experience of the elderly, society is less equipped to care for them (Sri-Arun, 2007; Wasee, 2006).

Realising that the tacit knowledge of elderly people is a cultural heritage as fragile as it is important, the Department of Public Welfare established the Bang Khae Home for Older Persons on $1^{\text {st }}$ June 1953 to provide essential care for elderly people with difficult economic and social circumstances. From these origins, a network of care homes and social welfare centres for older people was developed (Ministry of Social Development and Human Security, 2004). These care homes do not prevent changes to everyday lifestyle, physical health, mental health, society and the economy but they help elderly people cope with them. This investigation is designed to assess the state of institutional health care in four provinces of North-eastern Thailand, identify its problems and make suggestions for improvement.

\section{Methodology}

This qualitative investigation aims to outline the current state of institutional health care for the elderly in North-eastern Thailand (Isan) and suggest areas for development. The research period was from October 2012 to October 2013. Although there are many public and private institutions providing health care for the elderly in North-eastern Thailand, four locations were purposively selected for this investigation based on five criteria: they were in the Isan region, they were privately or publically sponsored, they were in active service, they continuously organised community activities for the elderly and they had developed a variety of physical and mental health services for elderly people. The locations chosen were:

2.1 Nursing Home, Division of Life Quality Promotion, Mahasarakham Provincial Administration Organisation (Government Sponsored)

The Mahasarakham Nursing Home was established on $24^{\text {th }}$ April 1986 and is located at 277 Moo 8, Mahasarakham-Wapi Pathum Road, Waeng Nang Sub-District, Mueang District, Mahasarakham (N. Srasan, personal communication, 2012). The objectives of the nursing home are to provide care for elderly people with 
difficulties, no relatives and nowhere to stay (S. Panta, personal communication, 2012). This is a government-sponsored institution.

\subsection{Ban Thammapakorn (Wat Muang) Nursing Home, Nakhon Ratchasima Province (Government Sponsored)}

Ban Thammapakorn (Wat Muang) Nursing Home was established in 1967 with the objectives to 'help elderly males and females over sixty years of age with difficulties, no relatives and nowhere to stay' (C. Wisetkwa, personal communication, 2012). The current location is 583 Potklang Road, Nai Mueang Sub-District, Mueang District, Nakhon Ratchasima. This is a government-sponsored institution.

\subsection{None Nong Wat 4 Elderly Community, Social Welfare Development Centre for the Elderly, Khon Kaen} Province (Privately Sponsored)

None Nong Wat 4 Elderly Community was established on the $20^{\text {th }}$ November 1995 to help provide care for elderly citizens. The community is located at 281 Lang Mueang Road, Nai Mueang Sub-District, Mueang District, Khon Kaen (S. Yangjaiyut, personal communication, 2012). This is a privately sponsored institution.

\subsection{Num Noi Sow Noi Community, Ban Samakee, Kalasin Province (Privately Sponsored)}

Num Noi Sow Noi Community was established in 2004 at the medical clinic of Ban Samakee, Moo 8, Don Sombun Sub-District, Yang Talat District, Kalasin (T. Sornsan, personal communication, 2012). The community has three main objectives: 'to uphold the legal rights and benefits of elderly people, to create spiritual concord and goodwill among its members and to allow its members to fulfill their potential...' (N. Supakdee, personal communication, 2012). This is a privately sponsored institution.

The research population was composed of people living at, working in or with a connection to the four institutions and their surrounding communities. The research sample was purposively selected and consisted of 70 individuals, divided into three groups (Table 1).

Table 1. Respondents used for data collection

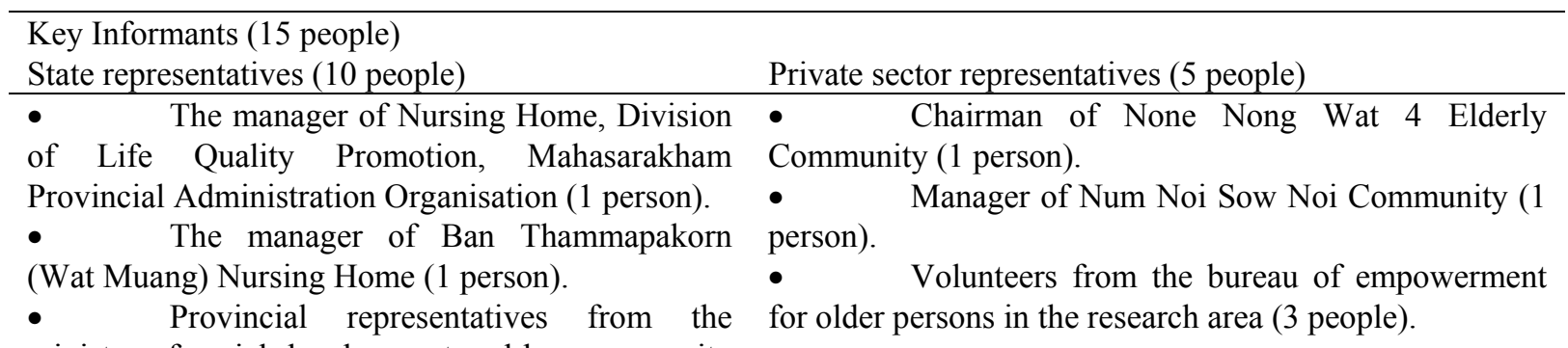
ministry of social development and human security (4 people).

- Directors from health promotion hospitals in the research area (4 people).

Casual Informants (40 people)

State representatives (20 people)

Private sector representatives (20 people)

- $\quad$ Employees at Nursing Home, Division of

Life Quality Promotion, Mahasarakham Provincial

Administration Organisation (5 people).

- $\quad$ Employees at Ban Thammapakorn (Wat

Muang) Nursing Home (5 people).

- Provincial social welfare officers (5

people).

- $\quad$ Provincial social development officers (5 people).

- $\quad$ Elderly people or representatives from Nursing Home, Division of Life Quality Promotion, Mahasarakham Provincial Administration Organisation (5 people).

- $\quad$ Elderly people or representatives from Ban

General Informants (15 people)

Thammapakorn (Wat Muang) Nursing Home (5 people).

- $\quad$ Elderly people or representatives from None Nong Wat 4 Elderly Community (5 people).

- $\quad$ Elderly people or representatives from Num Noi Sow Noi Community (5 people).

- $\quad$ Members of the general public with a personal interest in care for the elderly (10 people).

- $\quad$ Monks, students and academics interested in care for the elderly (5 people). 
Research tools used for data collection in the field were basic survey, observation, interview and focus group discussion. The survey was designed to gather general background information on the nursing homes and their residents. Both participant and non-participant observation techniques were used, with the research team attending and helping at nursing home activities during the participant stage. Two types of interview were carried out. Structured interviews were held with fixed questions according to the aims of the investigation and unstructured interviews were held to gather supplementary data. Focus group discussions were organized in each of the four research locations with eight to ten members of the research sample. All data was validated by methodological triangulation and analysed typologically according to the research aims, as detailed by Supang Chantavanich (2002). The results are here presented as a descriptive analysis.

\section{Results}

\subsection{The State of Institutional Health Care for the Elderly in North-eastern Thailand}

During this investigation, the four health care organisations were examined in five key areas: everyday lifestyle, physical health, mental health, society and economy. Below is given a description of findings at each institution.

\subsubsection{Mahasarakham Nursing Home}

The 100 male and female residents of the Mahasarakham Nursing Home come from all over North-eastern Thailand. 'We all live together happily and follow our daily routine. Every morning we wake, wash our faces and brush our teeth, exercise, wash, eat and then rest.' (A. Rapakdee, personal communication, 2013). The majority of residents have mobility problems and require assistance standing and sitting. These problems are primarily caused by aging but some residents have chronic illness, while others avoid exercise. 'As a group, I would say we don't recognise the importance of regular exercise and we regularly complain of tiredness, aches, pains or a fear of falling to avoid having to do it.'(S. Arom, personal communication, 2013). The care home organises medical and rehabilitation services for the residents in a designated first aid building. Professional resident nurses conduct regular health checks and implement treatment according to the advice of visiting doctors. For more serious conditions, the residents are sent for treatment at provincial hospitals. Additionally, there is a visiting medical clinic organised by the Division of Life Quality Promotion, Mahasarakham Provincial Administration Organisation. This is held every second Wednesday of the month from 13.30 to 16.30 ( $\mathrm{T}$. Nanamporn, personal communication, 2013). On top of deteriorating physical health, 'the mental state of many residents is fragile because they have been abandoned by their relatives or have no relatives remaining.' ( $\mathrm{N}$. Supakdee, personal communication, 2013). For those people who still have contact with family, 'worry, fear and a sense of burden occupy the mind' (A. Piwdaeng, personal communication, 2013).

'In the past, Isan society gave importance to the elderly because people believed in the value of experience and the responsibility of the family to take care of its senior members' (W. Tanusa, personal communication, 2013). There was a sense among the residents of Mahasarakham Nursing Home that the social importance afforded to elders had rescinded, and in some cases disappeared altogether. 'It's alright if you're a retired government official with a pension, a car, good health and free time to go where you like. It's another matter entirely if you're in poor health, have no money and no one to care for you.' (S. Sornsan, personal communication, 2013). The majority of residents at Mahasarakham Nursing Home fall into the latter category, either because of their health or their financial status. This causes their dependence upon a society they perceive as 'forgetful' (S. Peungluang, personal communication, 2013). Financial concerns are a particularly prominent factor in causing distress among the elderly residents of Mahasarakham Nursing Home. 'The majority of us worked in agriculture and manual labour. As soon as we retired, our regular exercise stopped, our health deteriorated and our income dried up.' (N. Pothom, personal communication, 2013).

\subsubsection{Ban Thammapakorn (Wat Muang) Nursing Home}

There are 96 over-60 residents at Ban Thammapakorn Nursing Home, of whom 27 are aged between 60 and 70, 32 are aged between 71 and 80, 31 are aged between 81 and 90 and six are aged over 91 . Everyday activities at the home include washing, eating, exercising, resting and meditation (P. Prayot, personal communication, 2013). Females are particularly diligent with their exercise routine and instructors will organise traditional dancing and stretching routines. Traditional herbalism is also used to help with physical health. 'We're given pandan juice to help with our heart and reduce thirst' (Y. Suriso, personal communication, 2013). Programs are organised to help residents maintain their health, such as the 'relaxing exercise program'. 'A responsible officer is placed in charge of every program and regular evaluation is conducted' (C. Wisetkwa, personal communication, 2013). From interviews, it was clear that local colleges and schools contributed to the health care by sending trainee nurses to gain experience in the nursing home. The mental health of patients does not depend solely on age. Other factors are responsible for mental health state, including lifestyle, diet and genetics. Some residents in their early sixties 
have a fragile state of mind, while others in their eighties and nineties are mentally strong. Many residents experience rapid mood swings, often born of frustration.

Socially, the majority of elderly residents worry and lack self-confidence when faced with other people. This is often a result of their loss of responsibility and social role. 'The newer generations are all so clever and streetwise. It makes me feel as though I know nothing about society, like my usefulness has passed' (Y. Suriso, personal communication, 2013). Ban Thammapakorn (Wat Muang) Nursing Home organised the national old people's day on $9^{\text {th }}$ April 2013 with Sub-Lieutenant Ranongrak Suwanchawee, president of the provincial administration organisation, as the chairperson. Mrs. Natcha Simanthammakul (personal communication, 2013), head of the Ban Thammapakorn (Wat Muang) Nursing Home, explained that 'this event provided an opportunity for children, relatives and friends of the elderly residents and people interested in the work of the nursing home to participate in activities, observe everyday life and make donations to the cause.' Activities such as a merit-making ceremony, elderly stage performances, entertainment, dancing and singing were welcome breaks from the everyday norm that enabled the elderly residents to interact with society. Importantly, 'they gave a chance to the senior citizens to show their talents and abilities, giving them confidence that they may continue as a part of society' (C. Kitdee, personal communication, 2013). The nursing home now has a large social network of 30 government and private institutions, including universities, schools and hospitals. Most residents at Ban Thammapakorn (Wat Muang) Nursing Home have no income. Consequently, the home organises its 'vocational remedy program', where the residents are encouraged to produce handmade goods, including flower decorations and floor mats. Merchants collect the finished products and sell them at local markets. 'This serves the dual purpose of generating extra income for the residents and enabling them to use their free-time productively' (N. Simanthammakul, personal communication, 2013).

\subsubsection{None Nong Wat 4 Elderly Community}

The majority of elderly people in the None Nong Wat Community live with their relatives but do not want to be a burden to their families. They are mostly healthy and strong-willed individuals who want to take care of themselves. As part of their everyday lives, they visit the temple and cooperate in temple ceremonies and services. 'The temple is a large part of or lives' (K. Lunsamrong, personal communication, 2013). The elderly community of None Nong are the chief advocates of the traditional local ceremony to sprinkle Buddha images with water and the primary source of inheritance. There are some members of the community who are too old and frail to take care of themselves. For these individuals, the family must take special care at meal and bath times. 'Their relatives must be strong and help them change their clothes, take care of their health and read them books' (T. Kongkeud, personal communication, 2013). There is a 30 minute exercise session every day to help the cardio-vascular systems of the community members. The community also provide dietary advice. There is a program to promote mental health, focused on four areas: mood, hobbies, the future and comfort. The program aims to put members in the right frame of mind or mood by encouraging them to take up suitable hobbies, think positively about the future and establish a network of friends. 'Having friends of the same age makes me feel as though I have worth and importance to society. It's a good feeling to have friends with whom I can exchange ideas and experiences' (K. Lunsamrong, personal communication, 2013).

The lack of mobility that comes with age and deteriorating health means that elderly people become more reliant upon their friends and relatives. This is a big reason for their alienation in society. The majority of people in the None Nong Community still have their families to care for them, which is a benefit of the large-family culture in North-eastern Thailand. The elderly community and their activities with the temple give members a network of peers with whom they can forge a new society so that they are unaffected by their decreasing sense of value in their old society. All elderly Thai citizens receive a pension and members of the None Nong Community are no different. 60-69 year-olds receive 600 baht per month, 70-79 year olds receive 700 baht per month, 80-89 year olds receive 800 baht per month and people over 90 receive 900 baht per month (S. Sri-Isan, personal communication, 2013). In addition, members of the None Nong Community are funded by their relatives and some use traditional knowledge to earn a supplementary income by producing handmade goods.

\subsubsection{Num Noi Sow Noi Community}

The Num Noi Sow Noi Community organises a number of everyday activities for its members. These include home health care and 'health through herbs', which aim to reduce weight through herbalism and exercise. The activities are overseen by the Motherland Volunteer Party. The community encourages its members to drink herbal juices found in the local area, including pandan, lemongrass and butterfly pea. Pandan is believed to help the cardio-vascular system and reduce thirst. The members are taught to mix their own pandan juice, as follows: 
Ingredients: one pandan leaf, one tablespoon of sugar, 16 tablespoons of water and half a teaspoon of salt.

Method: Wash the pandan leaf thoroughly and boil in the water. When the water comes to the boil, reduce the heat. Once the water is light green in colour, remove the leaf and add the sugar and salt. Increase the heat and boil for five minutes. Remove from the heat and pour into a mug. Drink while warm. (T. Patchima, personal communication, 2013)

Many members of the Num Noi Sow Noi Community have health problems, including diabetes, obesity, renal problems, osteoarthritis and stroke. An additional problem is poor oral hygiene which makes eating difficult and causes the selection of easy-to-chew foods, which often have high cholesterol. For these reasons, the community organises health programs, such as regular exercise and dental checks. There are also regular seminars to educate members in personal hygiene and health care. 'Representatives from the hospitals and clinics come to show us the right way to do things' (K. Poosomsri, personal communication, 2013). From observations it was clear that the care for elderly members of Num Noi Sow Noi Community from their relatives is thorough and personal. Nevertheless, there are some people who do not receive adequate care from their families because their relatives work away from home or they are in poor financial circumstances. These people must stay alone, often sad and forlorn but are invigorated by the work of Num Noi Sow Noi Community volunteers. In light of this, local residents organised an old people's day on $10^{\text {th }}$ April 2013.

There is active interest in the Num Noi Sow Noi Community among local society, which is visible in the support for activities. Local people have donated exercise mats, provided financial support for the old people's day and gave presents at a New Year's party. Num Noi Sow Noi Community also arranges many social activities for its members, including religious and cultural ceremonies such as regular merit-making services. 'There are also a number of social initiatives like communal cleaning programs and community care for sick members' (K. Pooseekiaw, personal communication, 2013). Aside from their state pension, the elderly members have little income unless it is provided by their families. The community organises a savings scheme, whereby members save one baht per day. This serves the dual function of providing members with savings in times of hardship and teaches them the importance and skill of saving money. 'It also shows us that we do have funds, if we use them wisely' (B. Poobuntong, personal communication, 2013).

\subsection{Problems with Elderly Healthcare}

Many residents have no family or the relatives they do have are prevented from helping by poverty. The elderly people are left lonely. This makes their lives difficult because they are in poor health and cannot help themselves. 'The biggest problem in the everyday life of residents is their dependence on others. There are few members of Mahasarakham Nursing Home who can help themselves' (P. Poomjaroen, personal communication, 2013). They find it difficult to move and cannot join in with activities due to poor health. Everyday life can be difficult because of a lack of funding and sponsorship. 'Even though the government provides a state pension, the costs of nursing care and everyday life are so high that elderly people don't have enough money for a comfortable everyday life' (P. Sudjai, personal communication, 2013). One of the biggest reasons for health deterioration is the diet of elderly people, who are reluctant to modify their traditional diet. 'There is not enough knowledge in the community to stimulate a change' (K. Somsri, personal communication, 2013). This is a particular problem among community members who still reside with their families and adopt the same lifestyle as their relatives.

The effects of old age are the biggest obstacle for elderly people in institutional nursing homes. Most physical health problems are caused by diabetes, high blood pressure and depression, which are all chronic conditions and require regular consultancy with a doctor. The elderly residents find it difficult to travel and, as there is a limited number of officers provided by the care homes to help with transportation, many residents do not receive the treatment they require. Residents in all four institutions believe that poor health leads to low confidence. Also, if the residents are ill, they cannot participate in community activities or exercise and feel alienated from society. There is the additional frustration and shyness caused by the inability to eat and go to the toilet independently. 'All this regular medical care has a significant effect on mental health and the onset of depression, which in turn causes physical problems' (N. Patchima, personal communication, 2013). The two are interrelated.

Nursing home residents come from many different places and are required to adapt culturally, which can make social integration difficult for some. During participant observation, the research team noticed a common complaint among residents was how much they missed their homes and previous lifestyle. This nostalgia is accompanied by insomnia, anxiety and pessimism and can lessen the unifying impact of group activities. 'The caregivers must be very strong individuals because they have to put on a positive face at all times' (B. Janpirom, personal communication, 2013). This is difficult given the fluctuating and often unpredictable mood swings in many residents. One of the principal concerns among elderly people is a fear of death. This is no different in 
institutional care homes. 'I think the worry is that no-one will manage our funeral and pray for our souls' (S. Suttiyo, personal communication, 2013).

The role of elderly people in society decreases with age because of personal changes and social pressures, such as health problems, generational language gaps and diet. This diminishing role means that older people are less inclined to participate in social activities. The younger generations are perceived as negligent towards the plight of the elderly. 'Some families work away and leave their parents and grandparents with a telephone. If there is an accident, how is the telephone going to help?' (Y . Ratanakunsat, personal communication).

Many of the elderly residents observed during this investigation enjoy playing the lottery. 'As they do not have enough funds to gamble, they borrow from friends at a high interest rate of 10-20 percent, which they agree to pay when their relatives visit or give them money' (P. Kamkuna, personal communication, 2013). In fact, almost all monetary problems are felt by elderly people with no sponsorship from relatives. The government pension is not enough to match the high cost of living and if the residents do not have another source of income they will suffer. 'If they don't have enough income, elderly people will sacrifice nursing care and all its health benefits to go to work, which hurts them physically and mentally in the long term' (S. Sri-Isan, personal communication, 2013). For care homes in rural areas, the economic strain on residents is lower due to the lower cost of living. The only institution with no economic problems among its members is Ban Thammapakorn Nursing Home. The reason for the good control of economic status among residents is the vocational remedy program, which allows each member to earn a living.

Respondents were asked to make suggestions for the development of institutional health care for the elderly that could improve the current situation and solve existing problems. Results from interviews and focus group discussions are summarized below (Table 2).

Table 2. The development of institutional health care for the elderly

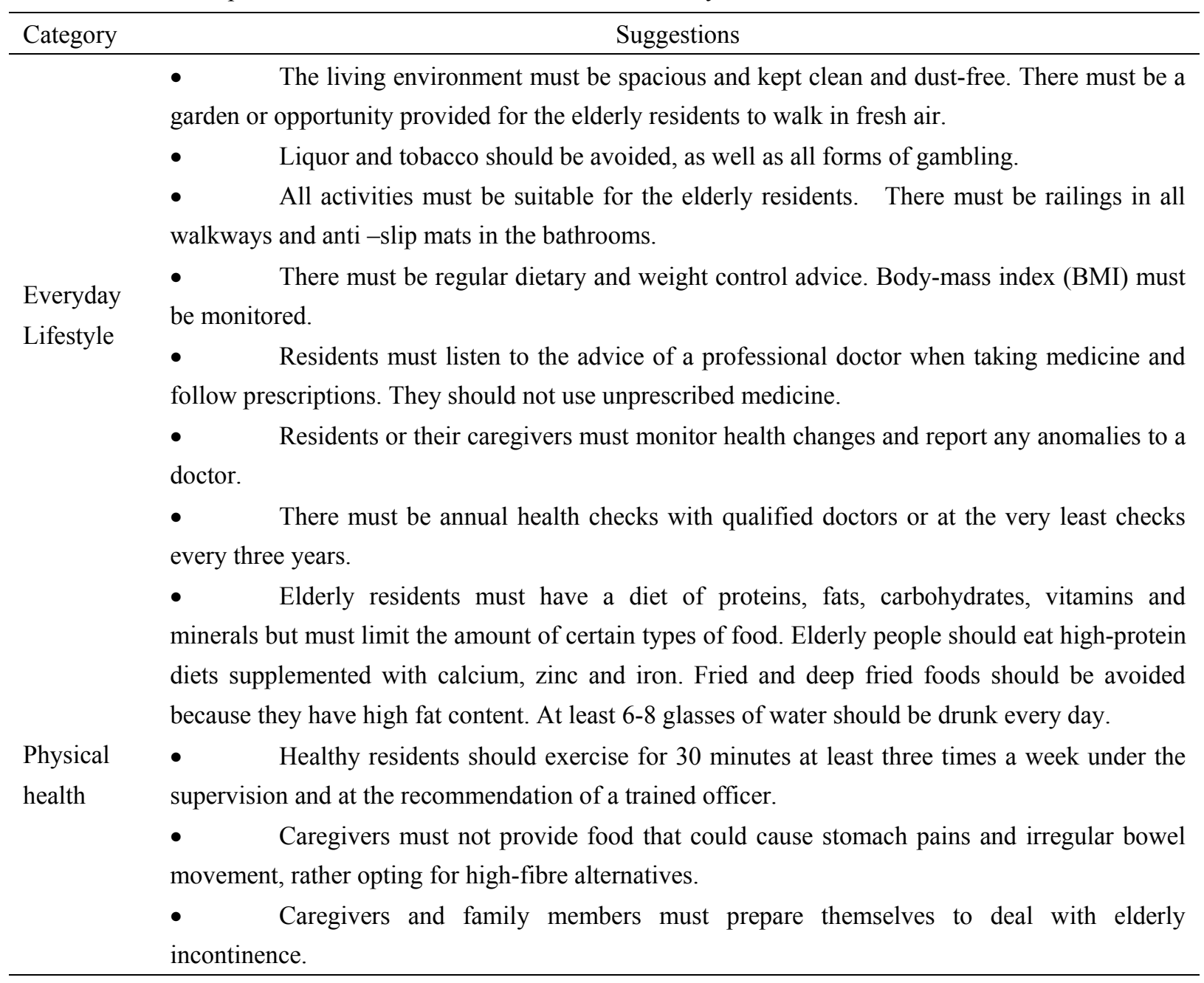




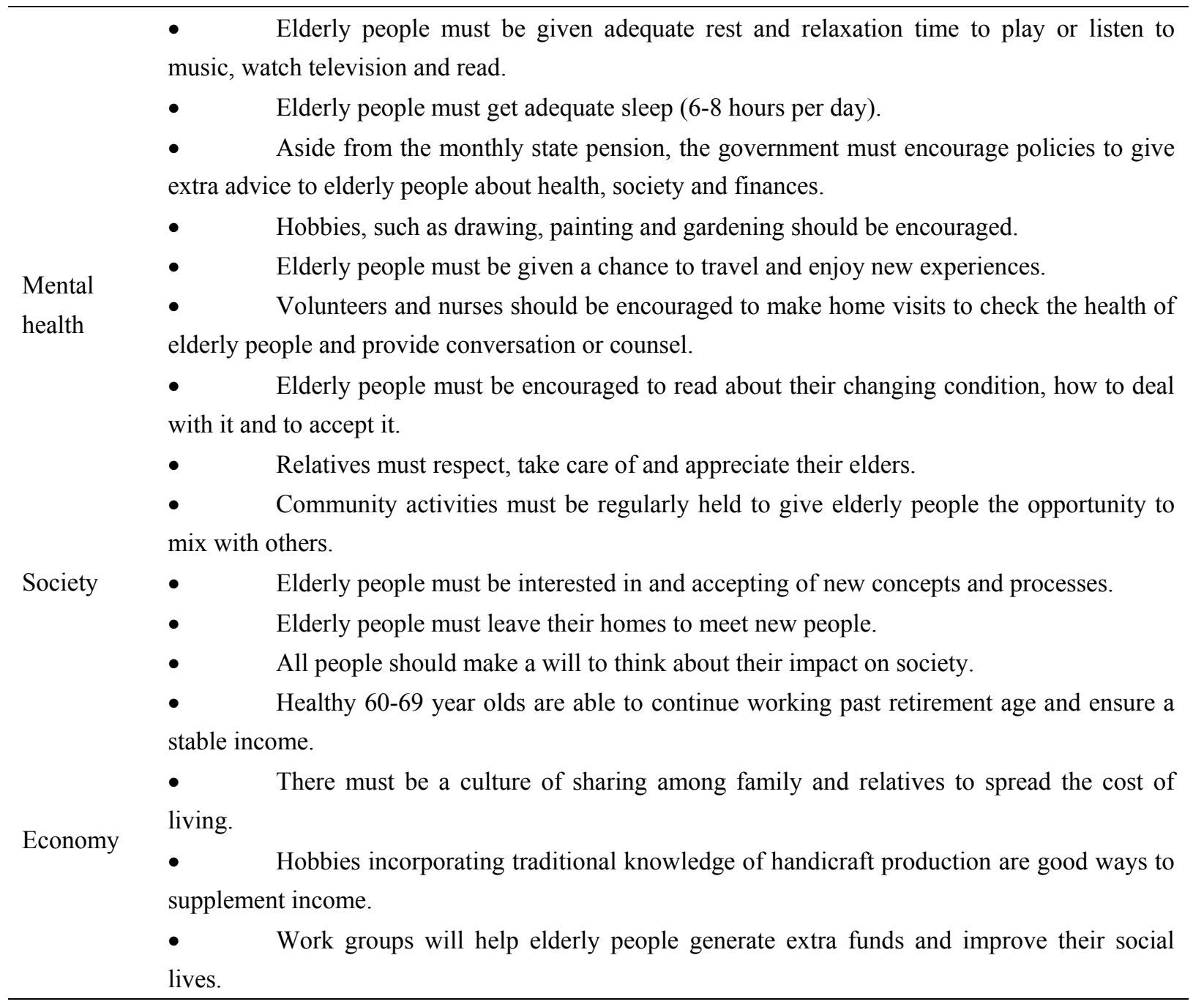

\section{Discussion}

This investigation found that the government provides experts to advise elderly communities about their diet. This is clearly of benefit because the traditional lifestyle of elderly people in North-eastern Thailand is based on glutinous rice and water, which provide energy throughout the day but do not constitute a complete and balanced diet. In later life, and especially in a nursing home, it is important for people to eat a well-balanced and nutritious diet (Weerakul, 2003). In addition to a good diet, regular exercise is important for maintaining the strength of elderly people. Despite the need to keep fit, over-exertion is a concern and trained officers must supervise all nursing home exercise activities. Yuttana Busanet (2005) found that elderly people in Roi Et province were in poor general health and suffered from back pain, hip pain and dizziness as a direct result of inadequate exercise. Busanet identified community exercise programs as a possible solution.

It is necessary for elderly people to reconsider the importance of their physical and mental health, which begins to change from the age of 50 onwards (Limchai-Arunrueang, 2003). To do this, they must receive support from the public and private sector (Arunwet, 2007). Support may be in the form of caregivers or self-help training but there is a larger network of people to be relied upon: relatives, neighbours, professional officers and volunteers (Yodpet, 2006). Rest and relaxation are just as important as exercise and can be achieved in a number of ways but must be promoted by the institutions. The care network plays a larger role for the elderly person by creating a sense of value and importance. The symbolic interaction theory of George Mead described how society is constructed by human interpretation of events and behaviour (Blumer, 1986; Mead, 1934). By showing respect for, and interest in elderly people, relatives, neighbours, professional officers and volunteers thus give them a social role and worth (Chantachon, 2010). It is the aim of elderly people to be or become a pillar of the community and act as a source of knowledge for younger generations (Srijakkot, 2007). For this reason, community activities are extremely important for society as they encourage a positive commitment from young 
people to help and respect their elderly friends and relatives. In a study of elderly health care in Northern Thailand, Caffrey (1991) found that elderly people cared for by their relatives were in better condition than those who had been neglected or abandoned.

Work schemes, such as the vocational remedy program of Ban Thammapakorn Nursing Home, are fantastic ways to generate additional income for nursing home residents and encourage elderly people to take up new hobbies, learn new skills and encounter new experiences. If work groups can be trained in marketing, production and sales by the government then their income and quality of life will increase (Daenseekaew et al., 2001).

Further research is required to implement the suggestions made by respondents during this investigation to determine their effectiveness. It would also be beneficial for future study to examine the extent of community participation in elderly health care and determine a method to enhance public interest. Moreover, this study found economic status to be a crucial factor in the everyday lifestyle, physical health, mental health and social status of elderly people. For this reason, research into the economic status of elderly people must be expanded. Through implementation of the suggestions made during this investigation, it is hoped that society may become more equipped to care for elderly people and the valuable cultural heritage they provide.

\section{References}

Arunwet, T. (2007). A method of developing elderly care in accordance with the 2007 social strategy of the ministry of social development and human security [in Thai]. Retrieved from www.m-society.go.th/article _attach/8937/11107.doc

Blumer, H. (1986). Symbolic interactionism: Perspective and method. Berkeley, CA: University of California Press.

Busanet, Y. (2005). Health problems among elderly people in Roi Et Province [in Thai]. PhD thesis, Mahasarakham University, Mahasarakham, Thailand.

Caffrey, R. (1991). Family caregiving to the elderly in Northeast Thailand: Changing patterns. PhD thesis, University of Oregon, Eugene, OR, USA.

Chantachon, S. (2010). Inheritance of traditional knowledge regarding land, water and forest resources among the Kaleung ethnic group [in Thai]. Mahasarakham: The Research Institure of North-eastern Arts and Culture.

Chantavanich, S. (2002). Qualitative research method [in Thai]. Bangkok: Chulalongkorn University Press.

College of Population Studies, Chulalongkorn University. (2007). Families and care for the elderly [in Thai]. Retrieved from http://www.cps.chula.ac.th/pop_info/thai/nop7/aging/support1.html

Daenseekaew, S. et al. (2001). Communities helping communities with the development of quality of life among elderly people in rural areas of Northeast Thailand [in Thai]. Khon Kaen: Khon Kaen University.

Jessell, M. A. (2007). Activities, control, and health in older adults. PhD thesis, Northcentral University, Prescott Valley, AZ, USA.

Limchai-Arunrueang, S. (2003). Factors having an effect on the learning processes of the elderly and their quality of life [in Thai]. PhD Thesis, Srinakharinwirot University, Bangkok.

Mead, G. H. (1934). In C. W. Morris (Ed.), Mind, self, and society from the standpoint of a social behaviorist. Chicago, IL: University of Chicago.

Ministry of Social Development and Human Security. (2004). Royal bill for elderly people, 2003 [in Thai]. Bangkok: J.S. Printing.

Sasat, S. (2006). Nursing elderly people: Common problems and methods of care [in Thai]. Bangkok: Chulalongkorn University Press.

Srijakkot, J. (2007). Action towards the elderly: A case study in the context of Isan social culture [in Thai]. Khon Kaen: Khon Kaen University.

Wasee, P. (2006). State pension of government officials. Communications of the association for state pension of government officials [in Thai]. Nakhon Ratchasima: Suriya Syndicate.

Weerakul, T. (2003). Terminal care for the elderly in nursing homes [in Thai]. Bangkok: Thammasat University.

Yodpet, S. (2006). Elderly welfare: Concepts and methods of social care (2nd ed.) [in Thai]. Bangkok: Mr. Copy (Thailand). 


\section{Copyrights}

Copyright for this article is retained by the author(s), with first publication rights granted to the journal.

This is an open-access article distributed under the terms and conditions of the Creative Commons Attribution license (http://creativecommons.org/licenses/by/3.0/). 